\title{
Discrete Line Congruences for Shading and Lighting
}

\author{
J. Wang ${ }^{1}$, C. Jiang ${ }^{1}$, P. Bompas, J. Wallner ${ }^{2}$ H. Pottmann ${ }^{1,3}$ \\ ${ }^{1}$ KAUST, $\quad{ }^{2}$ TU Graz, $\quad{ }^{3}$ TU Wien
}
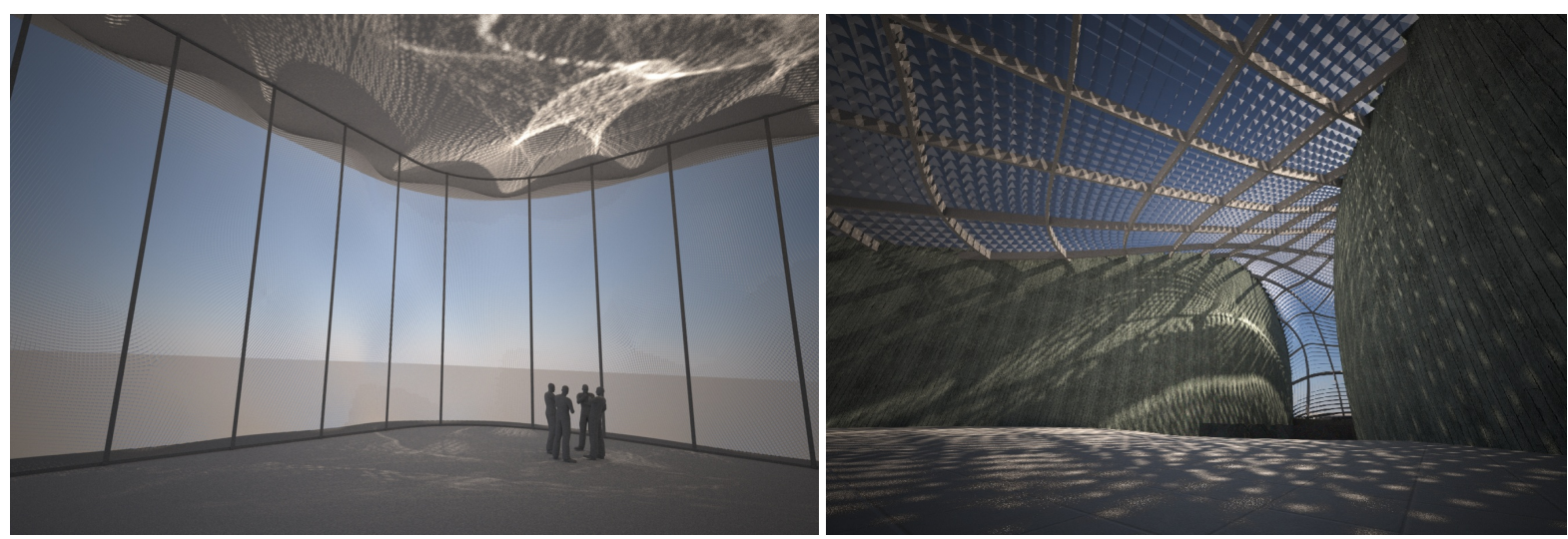

Figure 1: Line congruences constitute a basic geometric object in the computation of freeform shading and lighting systems for architecture. Our objects of study are discrete $2 D$ systems of straight lines which undergo optimization according to geometric requirements, followed by conversion to a non-manifold quad mesh with planar faces which is capable of blocking light, creating reflection patterns, or serving as part of the structure. In these two examples, all three of these optimization goals occur. Dark shadows are no error of rendering but the intended result of optimization; likewise the optimal placement of shading fins relative to light implies that the entire shading system can be shallow and transparent. For details see Figures 11 and 13.

\begin{abstract}
Two-parameter families of straight lines (line congruences) are implicitly present in graphics and geometry processing in several important ways including lighting and shape analysis. In this paper we make them accessible to optimization and geometric computing, by introducing a general discrete version of congruences based on piecewise-linear correspondences between triangle meshes. Our applications of congruences are based on the extraction of a so-called torsion-free support structure, which is a procedure analogous to remeshing a surface along its principal curvature lines. A particular application of such structures are freeform shading and lighting systems for architecture. We combine interactive design of such systems with global optimization in order to satisfy geometric constraints. In this way we explore a new area where architecture can greatly benefit from graphics.
\end{abstract}

Categories and Subject Descriptors (according to ACM CCS): Computer Graphics [I.3.5]: Computational Geometry and Object Modeling-

\section{Introduction}

This paper studies objects which implicitly are important parts of graphics and geometry processing in several ways, namely line congruences. These are 2-parameter families of straight lines, of which the light rays emanating from a point source are an example. Another example of much richer geometry is the system of lines which intersect a surface orthogonally, and which is closely tied to the curvature behaviour of that surface [Por94]. The geometry of smooth line congruences and their many relations to surfaces are well un- 
derstood. However there are only very few contributions to the topic of the present paper, which is discrete line congruences and their relations to discrete surfaces. We here propose a discrete version of line congruences and discuss their fundamentals as well as applications in shading and lighting systems in architecture.

Contributions. These include discrete line congruences based on triangle meshes $(\$ 2)$ and the important case of discrete normal congruences (\$3). Hitherto discrete congruences have mostly been studied in the form of their quadbased torsal parametrizations which could be interpreted as special quad-remeshings of triangle-based congruences. Both kinds are relevant for our paper, since our main application - shading systems - essentially is the same as a torsal parametrization. Our algorithmic contribution is a 2stage optimization procedure: We optimize a triangle-based congruence (\$4.1) such that quad-remeshing (\$4.2) yields the desired shading system, up to a bit of final optimization. Results (§4.3) and discussion ( $(5)$ conclude the paper.

Previous work. For an overview on line congruences with an emphasis on computing we refer to [PW01]. Design of congruences (with applications in mechanical engineering) has been studied by [GR98], who consider Bézier surfaces in an appropriate space of lines, thus modeling smooth congruences via a discrete control structure. Discrete normal congruences, with a computational framework for estimating focal surfaces of meshes with known or estimated normals, have been presented by [YYG*07]. There are some contributions to discrete line congruences in connection with special quad meshes (integrable systems), for which we refer to the monograph by [BS09]. They do not consider discrete versions of congruences, but rather discrete versions of the torsal parametrizations of congruences, which are also an important topic in the present paper. This theory has been first elaborated by [DSM00]. Of particular interest is the special case of discrete normal congruences, which lead to torsion-free support structures in architectural geom-

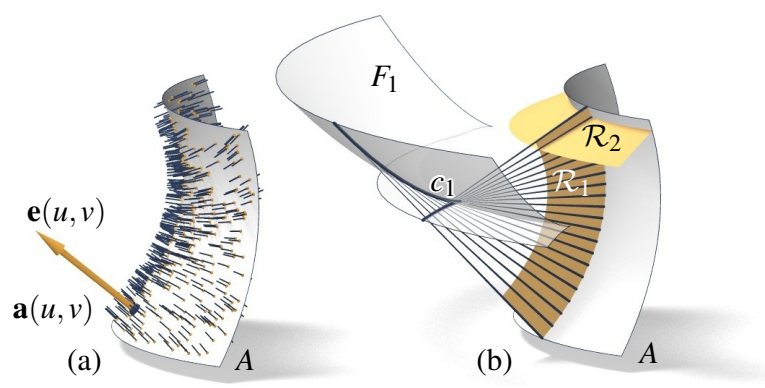

Figure 2: (a) A congruence $\mathcal{L}$ of lines $L(u, v)$ is described by a surface $A$, parametrized by $\mathbf{a}(u, v)$, and direction vectors $\mathbf{e}(u, v)$. (b) Developables $\mathcal{R}_{1}, \mathcal{R}_{2}$ contained in $\mathcal{L}$. The set of all regression curves $c_{i}$ of these developables makes up the focal sheets $F_{1}, F_{2}$ of the congruence (here only $F_{1}$ is shown). etry, special cases of which have already been considered in connection with meshes with specific offset properties $\left[\mathrm{LPW}^{*}\right.$ 06, PLW ${ }^{*}$ 07, PW08]. We should mention that also semidiscrete versions of these constructions are of importance in architecture [PSB* 08]. Finally refer to [KEN*12] and the references therein for light control in architecture.

\section{Line Congruences}

\subsection{Smooth line congruences}

We here recall a few facts from differential geometry. A line congruence $\mathcal{L}$ is a smooth $2 \mathrm{D}$ manifold of lines described locally by lines $L(u, v)$ which connect corresponding points $\mathbf{a}(u, v)$ and $\mathbf{b}(u, v)$ of two surfaces $A, B$. The vector $\mathbf{e}(u, v)=$ $\mathbf{b}(u, v)-\mathbf{a}(u, v)$ indicates the direction of the line $L(u, v) . \mathcal{L}$ is equivalently described by the volume parametrization

$$
\mathbf{x}(u, v, \lambda)=\mathbf{a}(u, v)+\lambda \mathbf{e}(u, v)=(1-\lambda) \mathbf{a}(u, v)+\lambda \mathbf{b}(u, v) .
$$

A ruled surface $\mathcal{R} \subset \mathcal{L}$ is described by functions $u(t), v(t)$ : A parametrization of such a ruled surface via parameters $t, \lambda$ is given by $\mathbf{x}(u(t), v(t), \lambda)$.

Torsal directions. In view of our applications we are especially interested in the developable ruled surfaces $\mathcal{R}$ contained in the congruence $\mathcal{L}$. Using subscripts for partial derivatives, and the symbol $[\cdot, \cdot, \cdot]$ for the determinant, the condition that $\mathcal{R}$ is developable reads $\left[\mathbf{e}, \mathbf{b}_{t}, \mathbf{a}_{t}\right]=0$ or equivalently $\left[\mathbf{e}, \mathbf{e}_{t}, \mathbf{a}_{t}\right]=0$ [PW01]. It expands to

$$
u_{t}^{2} \underbrace{\left.\mathbf{e}_{u}, \mathbf{a}_{u}, \mathbf{e}\right]}_{=: \gamma_{0}(u, v)}+u_{t} v_{t} \underbrace{\left(\left[\mathbf{e}_{u}, \mathbf{a}_{v}, \mathbf{e}\right]+\left[\mathbf{e}_{v}, \mathbf{a}_{u}, \mathbf{e}\right]\right)}_{=: 2 \gamma_{1}(u, v)}+v_{t}^{2} \underbrace{\left[\mathbf{e}_{v}, \mathbf{a}_{v}, \mathbf{e}\right]}_{=: \gamma_{2}(u, v)}=0 .
$$

For any fixed line $L(u, v)$ of the congruence, Equation (1) has up to 2 solutions $u_{t}: v_{t}$, which are called torsal directions. By integrating torsal directions one creates functions $u(t), v(t)$ which fulfill (1) and which describe developable surfaces $\mathcal{R}$ contained in $\mathcal{L}$ (see Fig. 2).

Example: Normal Congruences (see also §3). A classical example of a congruence is formed by the lines orthogonal to a surface $A$. In this case the normals along a principal curvature line constitute a developable ruled surface [Por94]. Thus such normal congruences always have torsal directions, namely the principal directions of $A$ (see Fig. 2).

REMARK (Undefined torsal directions). In the special case that $\mathcal{L}$ consists of the bundle of lines incident with a center, all ruled surfaces $\mathcal{R} \subset \mathcal{L}$ are cones (and thus developable) and all directions are torsal. It is important for us to know that such cases can occur, since optimization of congruences later in this paper may yield congruences close to a bundle, and defining a smooth frame field of torsal directions has to be assisted e.g. by a smoothness energy.

Focal points. We are especially interested in hyperbolic congruences where two torsal directions exist everywhere. 
Any line $L(u, v) \in \mathcal{L}$ is then contained in two developables $\mathcal{R}_{1}, \mathcal{R}_{2}$. It is known that this happens if and only if

$$
\left(\left[\mathbf{a}_{u}, \mathbf{e}_{v}, \mathbf{e}\right]+\left[\mathbf{e}_{u}, \mathbf{a}_{v}, \mathbf{e}\right]\right)^{2} \geq 4\left[\mathbf{e}_{u}, \mathbf{e}_{v}, \mathbf{e}\right]\left[\mathbf{a}_{u}, \mathbf{a}_{v}, \mathbf{e}\right] .
$$

To understand (2) we observe that among ruled surfaces, developables are characterized by having singular points on otherwise regular rulings (the curves of regression of Fig. 2). Thus, hyperbolicity implies that there exist singularities ( $f o-$ cal points) $\mathbf{x}(u, v, \lambda)$, where $\left[\mathbf{x}_{u}, \mathbf{x}_{v}, \mathbf{x}_{\lambda}\right]=0$, i.e.,

$$
\left[\mathbf{e}_{u}, \mathbf{e}_{v}, \mathbf{e}\right] \lambda^{2}+\left(\left[\mathbf{a}_{u}, \mathbf{e}_{v}, \mathbf{e}\right]+\left[\mathbf{e}_{u}, \mathbf{a}_{v}, \mathbf{e}\right]\right) \lambda+\left[\mathbf{a}_{u}, \mathbf{a}_{v}, \mathbf{e}\right]=0 .
$$

(3). This equation has solutions if and only if its discriminant is nonnegative, whence (2). For the converse statement and "singularities at infinity" we refer to [PW01]. Summing up, (2) holds $\Longleftrightarrow$ (3) has solutions $\Longleftrightarrow$ (1) has solutions.

Example: Congruences defined by affine mappings. Congruences defined by parametrizations of the form

$$
\mathbf{x}(u, v, \lambda)=\mathbf{a}_{0}+\mathbf{a}_{10} u+\mathbf{a}_{20} v+\lambda\left(\mathbf{e}_{0}+\mathbf{e}_{10} u+\mathbf{e}_{20} v\right)
$$

play an important in this paper. For fixed $\lambda=\lambda_{0}$, the mapping $\mathbf{x}\left(u, v, \lambda_{0}\right)$ parametrizes a plane $P_{\lambda_{0}}$. The affine mapping from $P_{\alpha}$ to $P_{\beta}$,

$$
\mathbf{x}(u, v, \alpha) \longmapsto \mathbf{x}(u, v, \beta),
$$

connects points which span the lines of the congruence. Focal points can be computed by (3). The following properties (see [PW01, Ex. 7.1.2] and Fig. 3) are important for us:

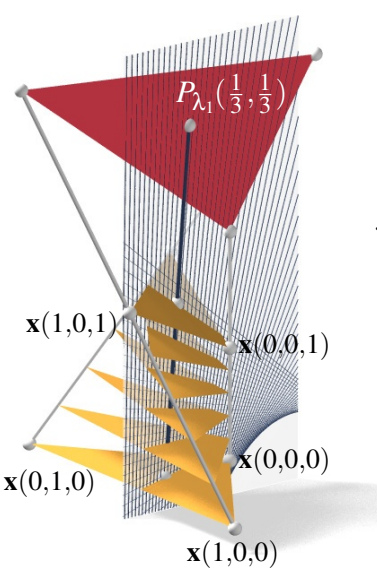

Figure 3: Congruence $\mathcal{L}$ defined by a "linear" volumetric parametrization $\mathbf{x}(u, v, \lambda)$. Planes $P_{\lambda}$ defined by $\lambda=$ const. are visualized as triangles. The red triangle $P_{\lambda_{1}}$ contains the ruling $L\left(\frac{1}{3}, \frac{1}{3}\right)$ and so the set of lines $P_{\lambda_{1}} \cap P_{\beta}, \beta \in$ $\mathbb{R}$, constitutes a developable $\mathcal{R} \subset \mathcal{L}$ through that ruling.

1. Each line $L=P_{\alpha} \cap P_{\beta}$ is contained in $\mathcal{L}$

2. The lines $P_{\alpha} \cap P_{\beta}$ with $\alpha$ fixed, constitute a developable surface $\mathcal{R} \subset \mathcal{L}$ which is planar and contained in $P_{\alpha}$ (in general, it is the tangent surface of a parabola).

We are going to make use of these congruences in the next subsection, when we consider discrete congruences defined by a correspondence between triangle meshes.

\subsection{Congruences defined over triangle meshes}

Let us define discrete congruences by means of two combinatorially equivalent triangle meshes $A, B$ with vertices $\left\{\mathbf{a}_{i}\right\}$
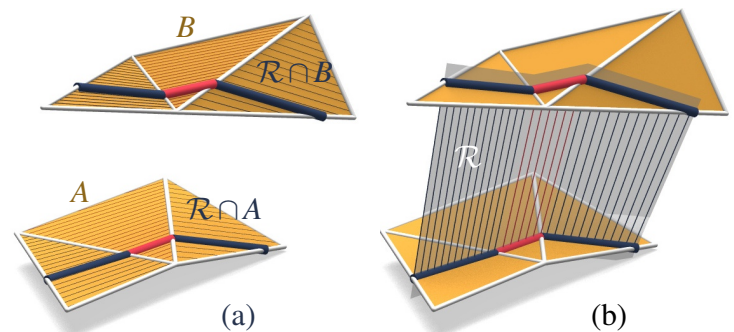

Figure 4: Piecewise-linear correspondence of meshes $A, B$ defining a piecewise-smooth congruence $\mathcal{L}$. (a) Integrating torsal directions yields corresponding polylines $A$ and $B .(b)$ Connecting corresponding points of those polylines yields a piecewise-flat (and thus developable) surface $\mathcal{R} \subset \mathcal{L}$.

and $\left\{\mathbf{b}_{i}\right\}$. The correspondence $\mathbf{a}_{i} \longleftrightarrow \mathbf{b}_{i}$ defines, via linear interpolation, correspondences between corresponding faces $\mathbf{a}_{i} \mathbf{a}_{j} \mathbf{a}_{k}$ and $\mathbf{b}_{i} \mathbf{b}_{j} \mathbf{b}_{k}$. Connecting corresponding points then yields a congruence $\mathcal{L}$ which is composed of pieces of the congruences studied in the example above. For each pair of corresponding triangles we let

$$
\mathbf{e}_{i}=\mathbf{b}_{i}-\mathbf{a}_{i}, \quad \mathbf{a}_{i j}=\mathbf{a}_{i}-\mathbf{a}_{j}, \quad \mathbf{e}_{i j}=\mathbf{e}_{i}-\mathbf{e}_{j}
$$

and obtain a volumetric parametrization of the type described in the example above:

$$
\begin{aligned}
& \mathbf{x}(u, v, \lambda)=\mathbf{a}(u, v)+\lambda \mathbf{e}(u, v), \\
& \mathbf{a}(u, v)=\mathbf{a}_{i}+u \mathbf{a}_{j i}+v \mathbf{a}_{k i}, \quad \mathbf{e}(u, v)=\mathbf{e}_{i}+u \mathbf{e}_{j i}+v \mathbf{e}_{k i} .
\end{aligned}
$$

Here $u, v$ run in the triangular domain $u, v, 1-u-v \geq 0$. In each point of a triangle we may now use Equations (1) and (3) to compute torsal directions and focal points.

In order to get a developable $\mathcal{R}$ contained in the congruence $\mathcal{L}$, we pick an initial point in a face and integrate the torsal directions from there. Property 2 above shows that as long as we stay within a face, the torsal directions integrate along straight lines (see Figure 4). When stepping over an edge from one triangle into the next one there are up to two possible torsal directions to continue, and we choose the one which has minimal deviation from the previous one. This procedure yields polylines $\mathcal{R} \cap A$ and $\mathcal{R} \cap B$, which subsequently span the developable $\mathcal{R}$, and which are considered a discrete representation of the developable $\mathcal{R}$.

\subsection{Congruences defined over quad meshes}

Algorithms on meshes frequently have the aim that their results depend as little as possible on the meshing but rather on geometric properties of the underlying assumed smooth shape which is approximated by the mesh. Sometimes however the mesh has regular combinatorics and the actual mesh polylines play an important role. This section, changing from triangle-based congruences to quad-based ones, performs exactly this change of viewpoint. Similar to $\$ 2.2$, assume combinatorially equivalent quad meshes 

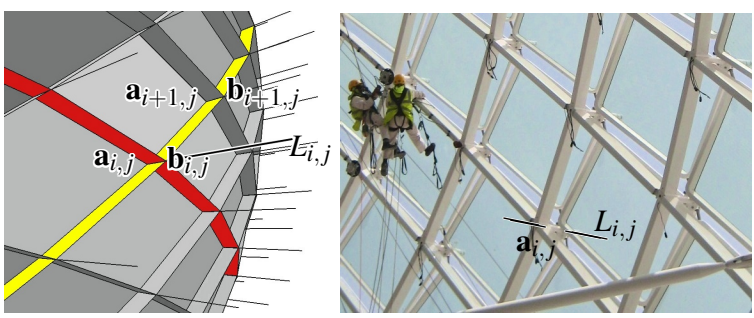

Figure 5: A torsal discrete congruence $\mathcal{L}$ is defined by connecting corresponding vertices of quad meshes $A, B$ where corresponding edges are co-planar; creating discrete developables (red and yellow) along mesh polylines. This congruence has been used for the Yas Marina hotel, Abu Dhabi (right) to create a torsion-free support structure.

$A, B$. We could imitate the construction of $\$ 2.2$ and define a piecewise-smooth congruence by bilinear interpolation within faces, but this does not lead to new insights. We therefore do not pursue this direction and reserve quad combinatorics for the treatment of torsal parametrizations of congruences:

DEFINITION 1. A parametrization $L(u, v)$ of a congruence is torsal, if the ruled surfaces defined by $u=$ const. are developable, and so are the ones defined by $v=$ const.

A discrete torsal parametrization is defined by a direct analogy: A discrete ruled surface (a sequence of lines) is developable if successive lines are co-planar (see colored ruled surfaces in Figure 5). Using this notion, we define:

DEFINITION 2. A line congruence $\left\{L_{i, j}\right\}$ of regular quad combinatorics is a torsal parametrization, if the ruled surfaces defined by $i=$ const. are developable, and so are those defined by $j=$ const.

Application: Torsion-Free Support Structures. Figure 5 shows a steel construction where prismatic beams follow the edges of a mesh, denoted by $A$, with regular quad combinatorics. $A$ has the additional property that each vertex $\mathbf{a}_{i, j}$ is equipped with a straight line $L_{i, j}$ such that for each beam adjacent to that vertex, the central symmetry plane of the beam contains $L_{i, j}$. Obviously this happens if and only if the lines $L_{i, j}$ constitute a discrete torsal parametrization.

Such constructions play an important part in the geometry of freeform architecture. We give them the name under which they are usually referred to in this context:

DEFINITION 3. A torsion-free support structure consists of combinatorially equivalent meshes $A, B$ such that corresponding edges are co-planar but do not coincide (in case of regular quad combinatorics, lines connecting corresponding vertices of $A, B$ constitute a torsal parametrization).

Doliwa et al. [DSM00], who first studied discrete torsal parametrizations in depth, use the word conjugacy for the relation between the mesh $A$ and the congruence $\mathcal{L}$. Previous work on torsion-free support structures was in the context of meshes with planar faces: [PLW*07] treat support structures in the context of architectural geometry. Actually planarity of faces of $A$ is an unnecessary restriction, see Fig. 5. It is an aim of the present paper to study and compute support structures consisting of meshes $A, B$ with non-planar faces, and to use them for new purposes.

\section{Normal congruences}

\subsection{Smooth normal congruences}

We already mentioned normal congruences, which are formed by the surface normals of a smooth surface $A$ (see Figure 2). The volume parametrization corresponding to such a congruence $\mathcal{L}$ reads $\mathbf{x}(u, v, \lambda)=\mathbf{a}(u, v)+\lambda \mathbf{e}(u, v)$, where $\mathbf{a}(u, v)$ parametrizes the surface $A$, and $\mathbf{e}(u, v)$ is the unit normal vector field. Note that any constant-distance offset $A^{d}$ of $A$ defines the same congruence, with $\mathbf{a}^{d}=\mathbf{a}+d \mathbf{e}$, $\mathbf{e}^{d}=\mathbf{e}$, and $\mathbf{x}^{d}(u, v, \lambda)=\mathbf{x}(u, v, \lambda+d)$.

Remark (Relation to Surfaces). Properties of normal congruences correspond directly to properties of surfaces: Torsal directions of $\mathcal{L}$ correspond to principal directions of $A$ ( $\Longrightarrow$ torsal directions exist everywhere). A developable surface in $\mathcal{L}$ consists of the surface normals along a principal curvature line of $A(\Longrightarrow$ there are two families of developables which intersect at right angles; actually this characterizes normal congruences). The focal surfaces of $\mathcal{L}$ consist of principal curvature centers of $A$, so they are a surface analogue of the evolute of a curve [Por94].

A general congruence $\mathbf{x}(u, v, \lambda)=\mathbf{a}(u, v)+\lambda \mathbf{e}(u, v)$ might be the normal congruence of an as yet unknown surface $\mathbf{a}^{*}(u, v)$ with normal vectors $\mathbf{e}(u, v)$. In order to find out if this is the case, we write $\mathbf{a}^{*}(u, v)=\mathbf{a}(u, v)+\lambda(u, v) \mathbf{e}(u, v)$ and solve for $\lambda(u, v)$. If we restrict ourselves to $\|\mathbf{e}\|=1$ we get $\left\langle\mathbf{e}, \mathbf{e}_{u}\right\rangle=\left\langle\mathbf{e}, \mathbf{e}_{v}\right\rangle=0$, and the orthogonality conditions $\left\langle\mathbf{e}, \mathbf{a}_{u}^{*}\right\rangle=\left\langle\mathbf{e}, \mathbf{a}_{v}^{*}\right\rangle=0$ are equivalent to $\lambda_{u}=-\left\langle\mathbf{a}_{u}, \mathbf{e}\right\rangle, \lambda_{v}=$ $-\left\langle\mathbf{a}_{v}, \mathbf{e}\right\rangle$. This PDE has a solution if and only if the integrability condition $\lambda_{u v}=\lambda_{v u}$ holds, i.e.,

$$
\left\langle\mathbf{a}_{u}, \mathbf{e}_{v}\right\rangle=\left\langle\mathbf{a}_{v}, \mathbf{e}_{u}\right\rangle .
$$

\subsection{Discrete normal congruences}

The following definition takes up a property characterizing smooth normal congruences:

DEFINITION 4. A congruence $\mathcal{L}$ defined by a piecewise-linear correspondence of triangle meshes A, B is called normal, if torsal planes (spanned by ruling and torsal direction) in the barycenters of faces are orthogonal.

We consider corresponding faces $\mathbf{a}_{1} \mathbf{a}_{2} \mathbf{a}_{3}$ and $\mathbf{b}_{1} \mathbf{b}_{2} \mathbf{b}_{3}$ of $A, B$, with difference vectors $\mathbf{e}_{i}=\mathbf{b}_{i}-\mathbf{a}_{i}$ and project them onto a plane orthogonal to the line connecting their barycenters. 
This yields triangles $\overline{\mathbf{a}}_{1} \overline{\mathbf{a}}_{2} \overline{\mathbf{a}}_{3}$ and $\overline{\mathbf{b}}_{1} \overline{\mathbf{b}}_{2} \overline{\mathbf{b}}_{3}$ and vectors $\overline{\mathbf{e}}_{i}=$ $\overline{\mathbf{b}}_{i}-\overline{\mathbf{a}}_{i}$ (see Figure 6). A discrete analogue of (5) now is the following:

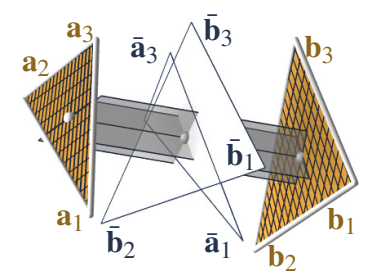

Figure 6: Congruences defined by corresponding meshes $A, B$ are normal if torsal planes in the barycenters of faces are orthogonal (here we show also the projection used by Prop. 1).

PROPOSITION 1. In the notation of the previous paragraph, meshes $A, B$ define a normal congruence $\Longleftrightarrow$ for each pair of corresponding faces, we have

$$
\left\langle\overline{\mathbf{a}}_{i j}, \overline{\mathbf{b}}_{i k}\right\rangle=\left\langle\overline{\mathbf{a}}_{i k}, \overline{\mathbf{b}}_{i j}\right\rangle,
$$

where $\overline{\mathbf{a}}_{i j}=\overline{\mathbf{a}}_{j}-\overline{\mathbf{a}}_{i}, \overline{\mathbf{b}}_{i j}=\overline{\mathbf{b}}_{j}-\overline{\mathbf{b}}_{i}$. This is equivalent to

$$
\left\langle\overline{\mathbf{a}}_{i j}, \overline{\mathbf{e}}_{i k}\right\rangle=\left\langle\overline{\mathbf{a}}_{i k}, \overline{\mathbf{e}}_{i j}\right\rangle,
$$

where $\overline{\mathbf{e}}_{i j}=\overline{\mathbf{e}}_{j}-\overline{\mathbf{e}}_{i}$. It is sufficient that these equations hold for at least one choice of indices $i \neq j \neq k$.

Proof. Corresponding points $\mathbf{a} \in A, \mathbf{b} \in B$ move in corresponding torsal directions $\mathbf{a}_{t}, \mathbf{b}_{t}$, resp., if and only if $\mathbf{b}-\mathbf{a}$, $\mathbf{a}_{t}, \mathbf{b}_{t}$ are coplanar, cf. the text above (1). With $\mathbf{a}, \mathbf{b}$ as barycenters of corresponding faces, this is obviously equivalent to linear dependence of $\overline{\mathbf{a}}_{t}, \overline{\mathbf{b}}_{t}$. When using the projection $\overline{\mathbf{a}}=\overline{\mathbf{b}}$ of barycenters as the origin of the coordinate system, there is a linear mapping $\alpha$ which maps corresponding points $\overline{\mathbf{a}}_{i} \mapsto \overline{\mathbf{b}}_{i}(i=1,2,3)$ as well as vectors $\overline{\mathbf{a}}_{t} \mapsto \overline{\mathbf{b}}_{t}$ (which are thus seen as eigenvectors of $\alpha$ ). This implies that normality is characterized by orthogonality of $\alpha$ 's eigenvectors, i.e., symmetry $\langle\mathbf{x}, \alpha(\mathbf{y})\rangle=\langle\alpha(\mathbf{x}), \mathbf{y}\rangle$ for at least 1 pair of linearly independent vectors $\mathbf{x}, \mathbf{y}$. This is exactly what is stated.

REMARK. It is easy to find conditions equivalent to (6). The following ones involve the difference of face centers,

$$
\mathbf{e}_{c}=\frac{1}{3}\left(\mathbf{b}_{1}+\mathbf{b}_{2}+\mathbf{b}_{3}\right)-\frac{1}{3}\left(\mathbf{a}_{1}+\mathbf{a}_{2}+\mathbf{a}_{3}\right),
$$

which indicates the direction of projection. We have

$$
\begin{aligned}
(6) & \Longleftrightarrow\left\langle\mathbf{a}_{i j} \times \mathbf{e}_{c}, \mathbf{b}_{i k} \times \mathbf{e}_{c}\right\rangle=\left\langle\mathbf{a}_{i k} \times \mathbf{e}_{c}, \mathbf{b}_{i j} \times \mathbf{e}_{c}\right\rangle \\
& \Longleftrightarrow\left\langle\mathbf{a}_{i j} \times \mathbf{e}_{c}, \mathbf{e}_{i k} \times \mathbf{e}_{c}\right\rangle=\left\langle\mathbf{a}_{i k} \times \mathbf{e}_{c}, \mathbf{e}_{i j} \times \mathbf{e}_{c}\right\rangle .
\end{aligned}
$$

\section{Applications and Algorithms}

The importance of torsion-free support structures for steel constructions has already been emphasized, see Fig. 5 and [PLW* $\left.{ }^{*} 7\right]$. We therefore demonstrate the capabilities of modeling with line congruences by means of another application, namely freeform shading and lighting systems.

Torsion-free support structures, which exhibit many planar quads, are well suited to function as shading elements themselves - see Figure 7. Their design is based on optimization of a line congruence $\mathcal{L}$, and subsequent extraction of a torsion-free support structure from $\mathcal{L}$ whose planes (i.e., torsal planes of $\mathcal{L}$ ) have the function of blocking light. It is very important that the combinatorics of the shading system is determined only in the second step, after optimization of the congruence has been performed.

A typical design objective for shading systems applications is the blocking of light by shading fins which correspond to torsal planes. We could require

(A) One family of $\mathcal{L}$ 's torsal planes is as orthogonal to incoming light as possible (so that those planes can function as shading fins of minimal possible width).

(B) As an alternative, the lines of $\mathcal{L}$ are as orthogonal as possible to the incoming light rays.

(A) achieves the goal of blocking light in a more obvious manner than (B), which indiscriminately moves all quads in the support structure in a position generally transverse to the light rays. Numerical experiments suggest that requiring (B) has the same effect as (A). Since it is simpler to implement we therefore employed (B) in most examples. The foundation of this observation is

PROPOSITION 2. For parallel light, generically $(\mathrm{B}) \Longrightarrow$ (A).

Proof. (В) $\Longrightarrow$ rulings of developables $\mathcal{R} \subset \mathcal{L}$ are orthogonal to light. Such developables can only be cylinders or planar. Generically not both families of developables in $\mathcal{L}$ are cylinders since then all rulings would be parallel. So at least one family of developables is planar, and torsal planes (being tangent planes of $\mathcal{R}$ ) are orthogonal to light rays.

This proof shows that congruences fulfilling (A) or (B) in an exact manner are rather special and will not occur in practice. Accordingly our examples achieve (A) or (B) only in a least squares sense. Further design objectives are:

(C) The user might prescribe individual lines of $\mathcal{L}$. Fitting a congruence to these data is not difficult [PW01], but in view of applications we want to do it such that $\mathcal{L}$ is hyperbolic and convertible to a torsal parametrization.

(D) Hyperbolicity is achieved by incorporating (6) into the optimization, making $\mathcal{L}$ more "normal".

(E) A user may prescribe torsal directions at selected locations of the mesh, in order to guide the appearance of the support structure later extracted from $\mathcal{L}$.

(F) A similar design objective is that incoming light is reflected in torsal planes in a prescribed way.

\subsection{Optimization of Mesh-based Congruences}

Most of the design objectives formulated above involve global optimization, and the following paragraphs show how to do that. We discuss how to optimize a congruence $\mathcal{L}$ defined by a fixed triangle mesh $A=(V, E, F)$ and a variable triangle mesh $B$ (we keep $A$ fixed since we are later remeshing anyway). Throughout this text, corresponding vertices of meshes $A, B$ are given by

$$
\mathbf{a}_{i} \quad \text { and } \quad \mathbf{b}_{i}=\mathbf{a}_{i}+\mathbf{e}_{i} \text { with } \quad\left\|\mathbf{e}_{i}\right\| \approx 1 .
$$




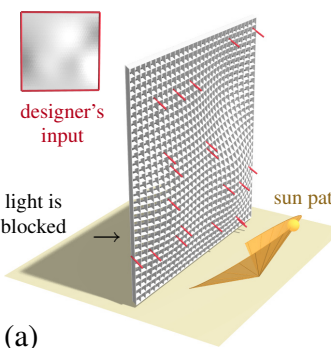

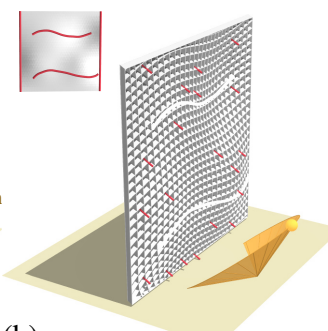

(b)

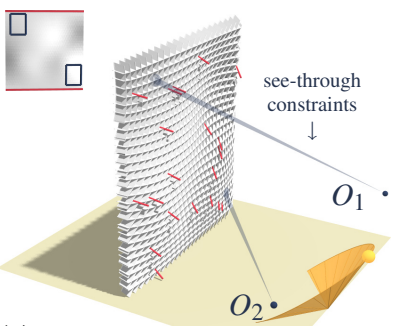

(c)

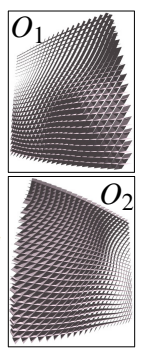

(d)

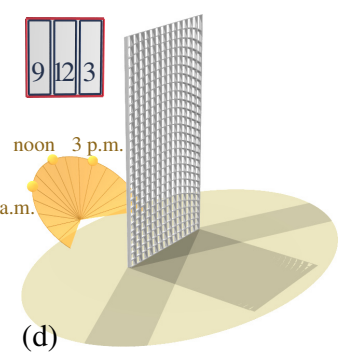

Figure 7: Shading systems with multiple constraints, computed by optimizing a line congruence (selected lines shown in red), subsequent conversion to torsal form and optimization towards planarity of shading fins. (a) Light is to be blocked, and and the boundary, torsal directions are to be aligned with the boundary. (b) Light is to be blocked, torsal directions are to be aligned with a user's design strokes. (c) Light is to be blocked, and in two selected areas of the facade, specified objects are to be visible (see inset figures at right for fish-eye views from $O_{1}$ and $O_{2}$ which verify this see-through constraint). (d) Here a truly flat facade is equipped with a shading system whose different parts block light emitted from different sun positions.

The lines $L_{i}$ of the congruence connect vertices $\mathbf{a}_{i}$ and $\mathbf{b}_{i}$. Restriction to unit vectors $\mathbf{e}_{i}$ yields simpler expressions for target functionals, at the cost of some degrees of freedom.

Contributions to target functionals. Below we list the components used to build the various target functionals for optimization which are employed in individual examples. Using an average edge length $\delta$, we appropriately normalize each term in order to make it scale invariant.

- Fairness. Assuming that $A$ is fair, we express fairness of the congruence in terms of the Laplacian of vectors $\mathbf{e}_{i}$ interpreted as a vector-valued function "e" on the mesh $A$ :

$$
f_{\text {fair }}=\frac{1}{|V|} \sum_{\mathbf{a}_{i} \in V}\left\|\Delta \mathbf{e}_{i}\right\|^{2}, \text { where } \Delta \mathbf{e}_{i}=\mathbf{e}_{i}-\frac{1}{\operatorname{deg} \mathbf{a}_{i}} \sum_{\mathbf{a}_{j} \sim \mathbf{a}_{i}} \mathbf{e}_{j} .
$$

- The normal congruence property. In the notation of (7), we penalize deviation from that property by

$$
f_{\text {norm }}=\frac{1}{\delta^{2}|F|} \sum_{\mathbf{a}_{i} \mathbf{a}_{j} \mathbf{a}_{k} \in F}\left(\left\langle\mathbf{a}_{i j} \times \mathbf{e}_{c}, \mathbf{e}_{i k} \times \mathbf{e}_{c}\right\rangle-\left\langle\mathbf{a}_{i k} \times \mathbf{e}_{c}, \mathbf{e}_{i j} \times \mathbf{e}_{c}\right\rangle\right)^{2} .
$$

- Hyperbolicity constraint. If our congruence is to have torsal planes, the discriminant condition (2) must hold everywhere. For practical purposes we require it for the barycenters of each face $\triangle=\mathbf{a}_{i} \mathbf{a}_{j} \mathbf{a}_{k}$. Using (4), it expands to

$$
\begin{aligned}
c_{\text {hyp }}(\triangle) & =\left(\left[\mathbf{a}_{i j}, \mathbf{e}_{i k}, \mathbf{e}_{c}\right]+\left[\mathbf{e}_{i j}, \mathbf{a}_{i k}, \mathbf{e}_{c}\right]\right)^{2} \\
& -4\left[\mathbf{e}_{i j}, \mathbf{e}_{i k}, \mathbf{e}_{c}\right]\left[\mathbf{a}_{i j}, \mathbf{a}_{i k}, \mathbf{e}_{c}\right] \geq 0 .
\end{aligned}
$$

- User-defined constraints. If the user specifies that the line $L_{i} \in \mathcal{L}$ should be parallel (resp., orthogonal) to a certain direction $\mathbf{d}_{i}$, we add appropriate linear combinations of

$$
f_{\mathrm{par}, i}=\left\|\mathbf{e}_{i} \times \mathbf{d}_{i}\right\|^{2}, \quad \text { resp. }, \quad f_{\text {perp }, i}=\left\langle\mathbf{e}_{i}, \mathbf{d}_{i}\right\rangle^{2}
$$

to the target functional, depending on the application. The constraint that the angle between $L_{i}$ and a user-specified vector $\mathbf{d}_{i}$ does not exceed a certain threshold is expressed as $\left\langle\mathbf{e}_{i}, \mathbf{d}_{i}\right\rangle-$ const. $\geq 0$ (here $\left\|\mathbf{e}_{i}\right\|=1$ is needed).

- Prescribing torsal directions and planes. If a user pre- scribes torsal directions in some part of the mesh, then we try to fulfill this wish for all faces $\triangle=\mathbf{a}_{i} \mathbf{a}_{j} \mathbf{a}_{k}$ which intersect that area of interest. We represent the required direction via a line segment $\mathbf{p}_{1} \mathbf{p}_{2} \subset \triangle$ containing the barycenter $\mathbf{c}=\frac{\mathbf{a}_{i}+\mathbf{a}_{j}+\mathbf{a}_{k}}{3}$. Fig. 4 makes it clear that $\mathbf{p}_{1} \mathbf{p}_{2}$ is torsal if

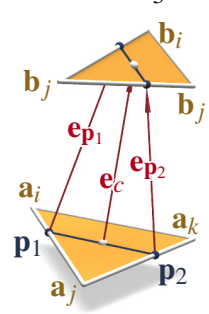
and only if the lines of $\mathcal{L}$ passing through the points $\mathbf{p}_{1}, \mathbf{p}_{2}, \mathbf{c}$ (indicated by vectors $\left.\mathbf{e}_{\mathbf{p}_{1}}, \mathbf{e}_{\mathbf{p}_{2}}, \mathbf{e}_{c}\right)$ are coplanar. A user wishing to prescribe an entire torsal plane must in addition specify its normal vector $\mathbf{n}$ which in particular is then orthogonal to $\mathbf{e}_{c}$. Summing up, for penalizing deviation from a desired torsal direction and plane, we use

$$
f_{\text {dir }}(\triangle)=\left[\mathbf{e}_{\mathbf{p}_{1}}, \mathbf{e}_{\mathbf{p}_{2}}, \mathbf{e}_{c}\right]^{2}, \quad f_{\text {plane }}(\triangle)=f_{\text {dir }}(\triangle)+\left\langle\mathbf{e}_{c}, \mathbf{n}\right\rangle^{2} .
$$

- Transversality of torsal planes. For applications it is often desirable that torsal planes intersect at right angles or nearly so. If we are optimizing towards a normal congruence, this property is automatic. Otherwise we use a condition of the form $c_{\text {ang }}(\triangle)>0$ which holds true if and only if the angle between torsal directions in the face $\triangle=\mathbf{a}_{i} \mathbf{a}_{j} \mathbf{a}_{k}$ does not fall below $\alpha$ ( $c_{\text {ang }}$ is a function taking arguments $\mathbf{a}_{i j}, \mathbf{a}_{i k}, \mathbf{e}_{i}$, $\mathbf{e}_{j}, \mathbf{e}_{k}, \alpha$ and is not printed here).

- Fairness of torsal directions is expressed in the smallness of jump in torsal planes when crossing an edge. Consider the line $L \in \mathcal{L}$ passing through the midpoint of an edge $\mathbf{a}_{i} \mathbf{a}_{j}$ of the mesh $A$ and project the adjacent triangles $\mathbf{a}_{i} \mathbf{a}_{j} \mathbf{a}_{k}$ and $\mathbf{a}_{i} \mathbf{a}_{j} \mathbf{a}_{l}$ orthogonally in direction $L$. This re-

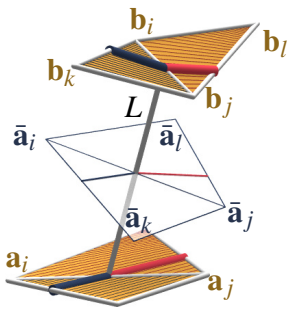
sults in vertices $\overline{\mathbf{a}}_{i}, \ldots$ "No jump" is expressed by the condition that torsal directions in the adjacent triangles project onto the same straight line. The same procedure can be applied to the mesh $B$. It is not difficult to verify that "no jump" is equivalently expressed by $\overline{\mathbf{a}}_{l}$ having the same barycentric coordinates w.r.t. $\overline{\mathbf{a}}_{i} \overline{\mathbf{a}}_{j} \overline{\mathbf{a}}_{k}$ as $\overline{\mathbf{b}}_{l}$ has w.r.t. $\overline{\mathbf{b}}_{i} \overline{\mathbf{b}}_{j} \overline{\mathbf{b}}_{k}$. An appro- 


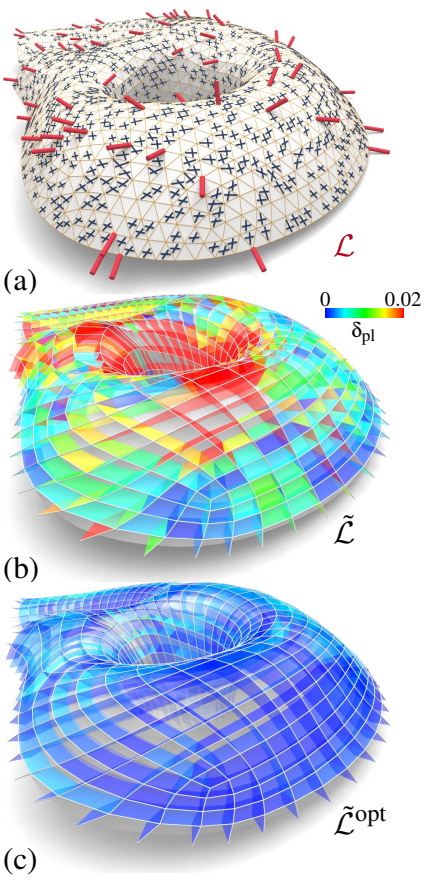

Optimize discrete line congruence $\mathcal{L}$ (red) defined by meshes $A, B$ (variables are vertices of $A, B$ ) and compute torsal directions in the faces of $A$ (blue)

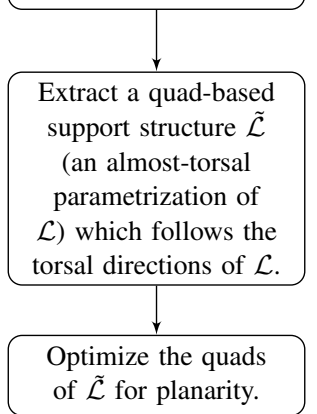

Figure 8: Flowchart of algorithm. For planarity measure $\delta_{\text {pl }}$ see Fig. 12.

priate sum of squares constitutes a fairness energy $f_{\text {fair/t }}$ and is added to the target functional as a regularizer.

Unconstrained and constrained optimization. We initialize optimization with vectors $\mathbf{e}_{i}$ which are estimates for normal vectors in vertices of $A$. We employ both unconstrained and constrained optimization. In the unconstrained case we minimize a combination of $f_{\text {fair }}, f_{\text {norm }}$, together with terms $f_{\text {dir }}(\triangle), f_{\text {plane }}(\triangle)$ and other terms which correspond to design specifications. This optimization problem is solved by a quasi-Newton method (limited-memory BFGS method [LN89]). The constraint $\left\|\mathbf{e}_{i}\right\|=1$ is enforced by simply re-normalizing all $\mathbf{e}_{i}$ 's after each round of iteration.

We also perform constrained optimization of the same kind of target functional, by adding user-defined constraints like $c_{\text {hyp }}(\triangle) \geq 0$ or $c_{\text {ang }}(\triangle) \geq 0$. We employ an augmented Lagrangian method to solve this constrained optimization problem. Again $\left\|\mathbf{e}_{i}\right\|=1$ is enforced by re-normalization.

\subsection{Conversion to Quad-based Torsal Form}

Converting the congruence $\mathcal{L}$ (defined by triangle meshes $A, B)$ to torsal form means finding a discrete torsal parametrization $\tilde{\mathcal{L}}$ (defined by quad meshes $\tilde{A}, \tilde{B}$ ) whose lines fit in the original congruence $\mathcal{L}$. The easiest method of conversion is to choose $\tilde{A}, \tilde{B}$ as respective remeshings of $A, B$, because then the edges of $\tilde{A}$ follow the torsal directions of $\mathcal{L}$ in $A$. The actual construction of $\tilde{\mathcal{L}}$ requires the two steps remeshing and optimization (see Figure 8).

Torsal Remeshing of Congruences. Still using the notation

from above, we first compute the frame field in $A$ which indicates the torsal directions of the congruence $\mathcal{L}$ (Fig. 8a). It is sufficient to compute the torsal directions by solving (1) for the barycenter of each face. We subsequently remesh $A$ to gain a mesh $\tilde{A}$ whose edges follow the frame field. This is a nontrivial task which we however do not consider a contribution of the present paper. We employed the method of [ $\left.\mathrm{LXW}^{*} 11\right]$, which is a version of mixed-integer quadrangulation [BZK09]. Once $\tilde{A}$ is known, we remesh $B$ by applying the correspondence $A \longleftrightarrow B$ to vertices of $\tilde{A}$, which yields vertices of $\tilde{B}$ (Fig. 8b).

Optimization of Support Structures. The preceding paragraphs show how to find corresponding meshes $\tilde{A}=(\tilde{V}, \tilde{E}$, $\tilde{F})$ and $\tilde{B}$ which represent an almost-torsal parametrization $\tilde{\mathcal{L}}$ of the congruence $\mathcal{L}$ (Fig. 8b). We optimize $\tilde{A}, \tilde{B}$ such that corresponding edges become co-planar:

$$
\tilde{\mathbf{a}}_{i}, \tilde{\mathbf{a}}_{j}, \tilde{\mathbf{b}}_{i}, \tilde{\mathbf{b}}_{j} \text {, co-planar, whenever } \tilde{\mathbf{a}}_{i} \tilde{\mathbf{a}}_{j} \in \tilde{E} .
$$

We wish to achieve this while retaining proximity of $\tilde{A}$ to the reference surface $A$, and likewise retaining proximity of $\tilde{\mathcal{L}}$ to the reference congruence $\mathcal{L}$. We therefore minimize

$$
\begin{aligned}
\tilde{f} & =\tilde{w}_{\text {plnt }} \frac{1}{\delta^{2}|\tilde{E}|} \sum_{\mathbf{a}_{i} \mathbf{a}_{j} \in \tilde{E}} \operatorname{dist}\left(\tilde{\mathbf{a}}_{i} \vee \tilde{\mathbf{b}}_{j}, \tilde{\mathbf{a}}_{j} \vee \tilde{\mathbf{b}}_{i}\right)^{2} \\
& +\tilde{w}_{\text {prox }} \frac{1}{|\tilde{V}|} \sum_{\tilde{\mathbf{a}}_{i} \in \tilde{V}}\left(\left\|\tilde{\mathbf{e}}_{i}-\tilde{\mathbf{e}}_{i, 0}\right\|^{2}+\left(\frac{\operatorname{dist}\left(\tilde{\mathbf{a}}_{i}, A\right)}{\tilde{\delta}}\right)^{2}\right), \\
& +\tilde{w}_{\text {fair }} \frac{1}{\tilde{\delta}^{2}|\tilde{V}|} \sum_{\tilde{\mathbf{a}}_{i} \in \tilde{V}}\left\|\Delta \tilde{\mathbf{a}}_{i}\right\|^{2} .
\end{aligned}
$$

Here the first summand (with weight $\tilde{w}_{\text {plnr }} \geq 0$, normalized by an average edge length $\tilde{\delta}$ ) penalizes deviation of quadrilaterals $\tilde{\mathbf{a}}_{i} \tilde{\mathbf{a}}_{j} \tilde{\mathbf{b}}_{i} \tilde{\mathbf{b}}_{j}$ from planarity by computing the sum of squares of distances of their diagonals.

The second summand (weighted with $\tilde{w}_{\text {prox }}$ ) penalizes deviation of the vectors $\tilde{\mathbf{e}}_{i}$ (indicating lines of $\tilde{\mathcal{L}}$ ) from their initial values $\tilde{\mathbf{e}}_{i, 0}$; and deviation of vertices $\tilde{\mathbf{a}}_{j}$ from the reference surface $A$. Here the symbol $\operatorname{dist}\left(\mathbf{a}_{i}, A\right)$ does not really mean the distance from $A$ (which is hardly efficiently computable), but an approximation of that distance by $\operatorname{dist}\left(\mathbf{a}_{i}\right.$, $\left.T_{i}\right)^{2}$, where $T_{i}$ is an estimated tangent plane of $A$ in the point which arises by closest-point projection onto $A$ of the position of $\mathbf{a}_{i}$ in the previous round of iteration. See Fig. 8c, and see Figure 12 for details on the choice of weights.

\subsection{Results}

We apply $\S 4.1, \S 4.2$ to shading systems with both planar and developable elements, and also to indirect lighting.

Shading Systems for Facades (Figure 7). In each of these examples a congruence $\mathcal{L}$ is optimized so that a torsionfree support structure extracted from it blocks the rays of the sun during the hottest parts of the day. The astronomical information necessary to perform such computations is easily obtainable, since the path of the sun throughout the year is known. For optimization we simply employ directions of 

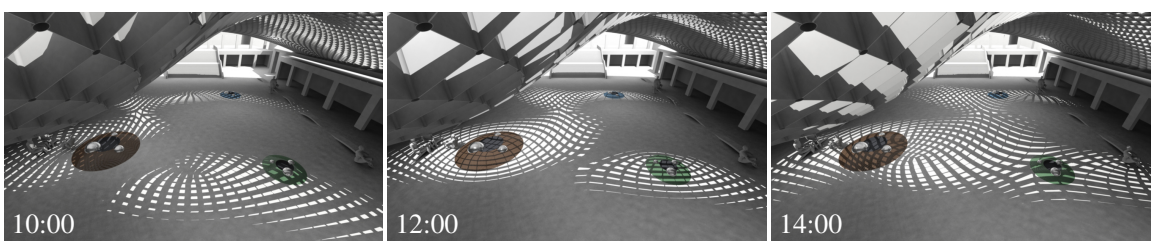

Figure 9: Selective Shading: Moving patterns generated by shading system optimized for blocking light at 12:00 except at designated areas.

light which correspond to the location of the sun during "hot" times like early afternoon in summer. If the depth of shading fins is made minimal, then obviously at other times the sun is not completely blocked. Note that these shading systems are "freeform" even if the underlying reference surface is not, such as in Figure 7d.

For Figure 7 in general, a design surface (referred to as "mesh $A$ " in our description of the optimization procedure) is equipped with a line congruence $\mathcal{L}$, which is initialized from surface normals of $A$ and is subsequently optimized using a target functional composed of $f_{\text {norm }}, f_{\text {fair }}$, and a linear combination of terms $f_{\text {perp }, i}$ (among other terms). The latter make lines of $\mathcal{L}$ orthogonal to the vector $\mathbf{d}_{i}=\mathbf{d}=$ const. which indicates the direction of light. Having computed $\mathcal{L}$, we perform quad remeshing guided by $\mathcal{L}$ 's torsal frame field, and subsequently optimize a torsion-free support structure.

For Figures $7 \mathrm{a}, 7 \mathrm{~b}$ the support structure is to be aligned with the boundary and a user's design strokes, so optimization uses terms $f_{\text {dir }}(\triangle)$ to achieve prescribed torsal directions for faces contained in a certain subset $F^{\prime} \subseteq F\left(F^{\prime}\right.$ is marked in red in small inset figures). In a similar manner the shading system of $7 \mathrm{~d}$ has been optimized. As an alternative to $f_{\text {perp }, i}$, here sun blocking is achieved using a linear combination of terms $f_{\text {plane }}(\triangle)$ which position torsal planes of the congruence directly orthogonal to incoming light.

Finally Figure 7c exhibits a shading system with the property that certain objects are visible through the shading system in designated areas (blue rectangles in inset figure). Optimization therefore has to make sure that for vertices in a subset $V^{\prime} \subseteq V$ the lines of $\mathcal{L}$ pass through prescribed target points $O_{i}$. This constraint is incorporated into our optimiza-

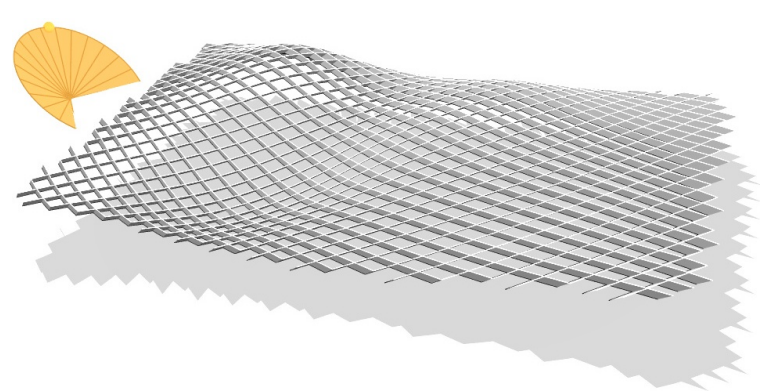

Figure 10: Creating full shade by thin developable strips, created by the application of subdivision+optimization to a shading system with planar faces. tion by augmenting the target functional with linear combinations of $f_{\mathrm{par}, i}$, for vertices in $V^{\prime}$. Such constraints could be used e.g. for ensuring that people in offices see a portion of the sky. InewFor optimizing the congruences $\mathcal{L}$ corresponding to Figure 7, we use the target functional

$$
\begin{aligned}
f & =w_{\text {norm }} f_{\text {norm }}+w_{\text {fair }} f_{\text {fair }}+w_{\text {fair } / \mathrm{t}} f_{\text {fair/t }} \\
& +w_{\text {perp }} \frac{1}{|V|} \sum_{\mathbf{a}_{i}} f_{\text {perp }, i}+w_{\text {dir }} \frac{1}{\left|F^{\prime}\right|} \sum_{\triangle \in F^{\prime}} f_{\text {dir }}(\triangle) \\
& +w_{\text {par }} \frac{1}{\left|V^{\prime}\right|} \sum_{\mathbf{a}_{i} \in V^{\prime}} f_{\mathrm{par}, i}+w_{\text {plane }} \frac{1}{|F|} \sum_{\triangle \in F} f_{\text {plnr }}(\triangle) .
\end{aligned}
$$

For constraints and the choice of weights see Figure 12.

Selective Blocking of Light (Figure 9). This architectural design is to give shade except for a designated area where shading fins are to be parallel to incoming rays. To create this example we proceed similar to Figure 7c: The base mesh represents the design surface, its normals initialize $\mathcal{L}$. A subset $V^{\prime} \subset V$ of vertices specifies the area where light should come through. The optimization uses the target functional (9), with the 'parallel' and 'perpendicular' terms given as

$$
\frac{w_{\text {par }}}{\left|V^{\prime}\right|} \sum_{\mathbf{a}_{i} \in V^{\prime}} f_{\text {par }, i}+\frac{w_{\text {perp }}}{\left|V \backslash V^{\prime}\right|} \sum_{\mathbf{a}_{i} \in V \backslash V^{\prime}} f_{\text {perp }, i} .
$$

( $f_{\mathrm{par}, i}, f_{\mathrm{perp}, i}$ involve the direction $\mathbf{d}_{i}=\mathbf{d}=$ const. of light).

Shading by Single-Curved Elements (Figure 10). A sequence of planar quadrilaterals is a discrete developable surface (see e.g. the red and yellow developables of Figure 5). This interpretation motivates us to apply a refinement procedure according to $\left[\mathrm{LPW}^{*} 06\right]$ to a torsion-free support structure in order to convert it into a system of smooth developables: we iteratively apply splitting, smoothing, and optimization towards planar faces. Applications are structures built from plywood or sheet metal, whose manufacturing depends on the developability property.

Indirect Lighting by Reflection (Figures 1, 11, 13). We extend our methods to indirect lighting by reflection. To guide a ray of light towards a prescribed direction, a bisector plane of the original ray and the reflected ray has to be used as a mirror surface. We therefore optimize a congruence $\mathcal{L}$ such that precomputed mirror planes become torsal planes. Figure 11 actually exhibits a 2 nd torsion-free supporting structure, which does have the function indicated by its name, namely a steel substructure aligned with the shading system (Fig. 11(d3)). It is based on a congruence $\mathcal{L}^{\prime}$ which is optimized simultaneously with $\mathcal{L}$. Alignment of $\mathcal{L}, \mathcal{L}^{\prime}$ means that torsal directions of $\mathcal{L}, \mathcal{L}^{\prime}$ coincide which is achieved by 

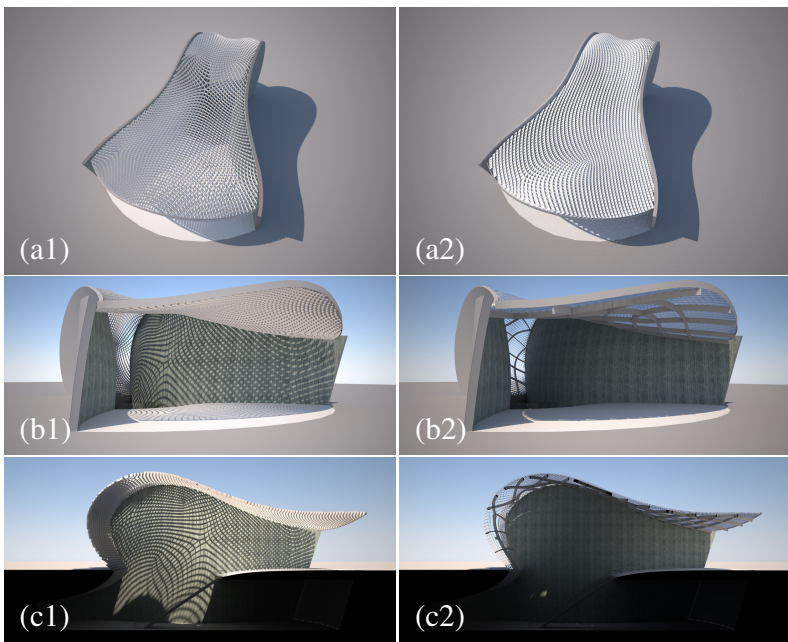

Figure 11: Shading and lighting systems for a subway entrance in London at noon, June 21. Rows a-d represent top view, front view, cross-section, and interior view, respectively. Columns 1-4 correspond to different shading systems extracted from a congruence $\mathcal{L}:$ In (a1)-(c1) $\mathcal{L}$ consists of the normals of the mesh A which represents the roof, so the support structure follows the principal curvature lines of $A$. There is no effective shading. In (a2)-(c2) $\mathcal{L}$ is optimized for blocking sunlight. In (a3)-(e3) $\mathcal{L}$ is optimized such that shading fins reflect sunlight towards the interior at an angle of 45 degrees, with (e3) illustrating reflected light rays. In (a4) (e4) the front part of the roof is optimized for shading, the rest for reflection.

augmenting the target function (9) by

$$
w_{\text {extra }} \cdot \frac{1}{|F|} \sum_{\triangle \in F}\left(\frac{1}{\delta^{2}}\left\|\left(\begin{array}{c}
\gamma_{0} \\
2 \gamma_{1} \\
\gamma_{2}
\end{array}\right)_{\mathcal{L}} \times\left(\begin{array}{c}
\gamma_{0} \\
2 \gamma_{1} \\
\gamma_{2}
\end{array}\right)_{\mathcal{L}^{\prime}}\right\|\right)^{2}
$$

( $\gamma_{i}$ are the coefficients of (1), evaluated in face barycenters).

\section{Discussion}

Implementation Details. Details on optimization for the examples contained in this paper are given in Figure 12. In particular we give the quality of planarity for each occurring torsion-free support structure. We found that planarity is mostly sufficient already after the extraction procedure of $\$ 4.2$ so no further optimization is needed. Timings are for a $2.4 \mathrm{GHz}$ dual core desktop with 6GB RAM.

Limitations. We generally found in our examples that we could successfully optimize congruences as desired. However it is not possible to fulfill our kind of geometric sideconditions for normal congruences. As a consequence, the contribution $f_{\text {norm }}$ to the target functional works as a regularizer and more importantly, it makes congruences hyperbolic and therefore usable for support structures. A general limitation of static systems which block or guide light from moving sources is, of course, that they are optimal only for

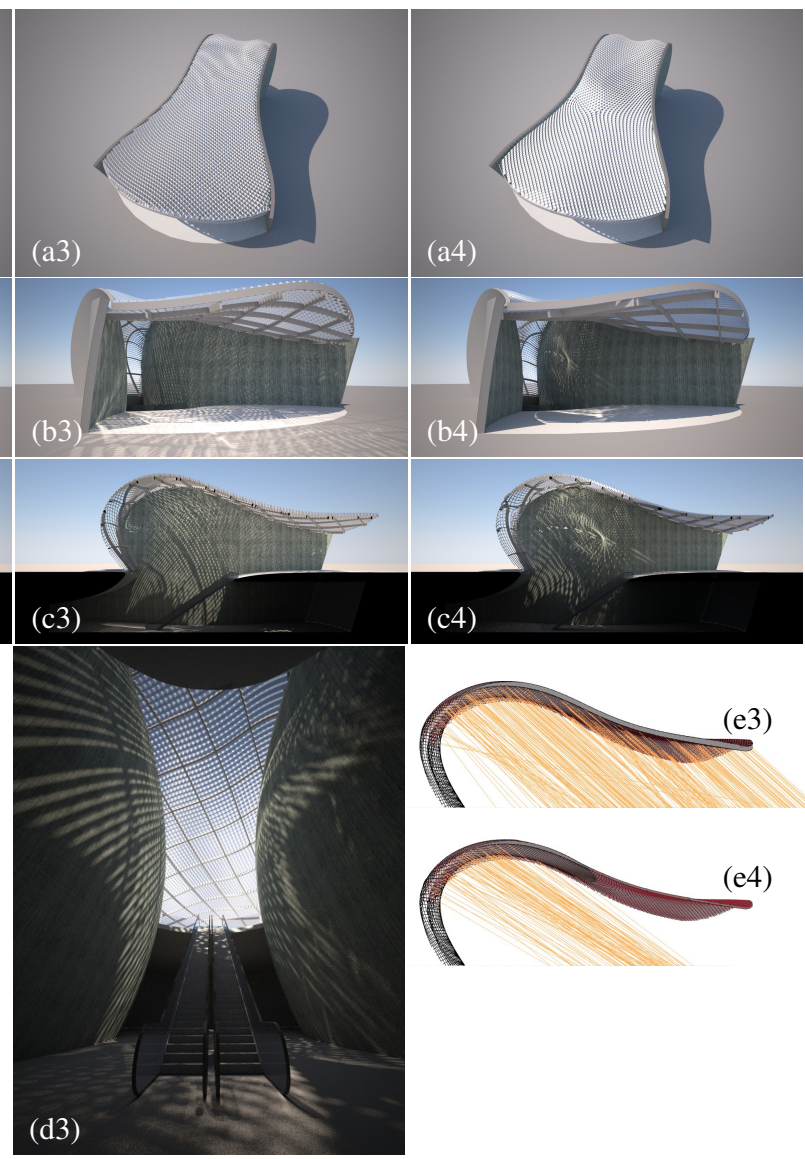

the few positions of the light source they have been optimized for; see Figures $7 \mathrm{~d}$ and 9.

Robustness. We take as evidence for robustness of our nonlinear optimizaton procedures that we could initialize congruences from lines orthogonal to the reference surface, even if the result of optimization is far from orthogonal. Experiments show that adding noise (uniformly distributed, up to maximum $\approx 50^{\circ}$ ) does not visibly influence the result.

Alternative Routes. We employ a two-step procedure: optimization of a congruence and subsequent extraction of a torsion-free support structure (which determines the combinatorics of the shading system). The separation into these two steps is essential: we found that the simpler method of directly optimizing vertex positions of a non-optimal shading system does not work. An alternative approach is to determine the orientation of torsal planes from the desired light pattern. For smooth congruences 1 family of torsal planes determines the congruence including the 2nd family of torsal planes (by differentiating twice). This method works in principle, but we found it not very robust.

Conclusion and Future Research. This paper demonstrates geometric basics and applications of discrete congruences, 
J. Wang, C. Jiang, P. Bompas, J. Wallner, H. Pottmann / Discrete Line Congruences for Shading and Lighting

\begin{tabular}{|c|c|c|c|c|c|c|c|c|c|c|c|c|c|c|c|c|c|c|c|c|c|}
\hline \multirow[t]{2}{*}{ Fig. } & \multicolumn{13}{|c|}{ optimization of congruence $\mathcal{L}$ and meshes $A, B$, cf. Equ. (9) } & \multicolumn{3}{|c|}{ extracting $\tilde{A}, \tilde{B}$} & \multicolumn{5}{|c|}{ optim. $\tilde{A}, \tilde{B}$, cf. (8) } \\
\hline & $|V|$ & $|F|$ & $T$ & $w_{\text {extra }}$ & $w_{\text {norr }}$ & $w_{\text {fai }}$ & $w_{\text {fair }}$ & $w_{\text {perp }}$ & $w_{\text {par }}$ & $w_{\text {plane }}$ & $w_{\text {dir }}$ & $c_{\text {hyp }}$ & $c_{\text {ang }}$ & $|\tilde{F}|$ & $|\tilde{E}|$ & $\delta_{\mathrm{pl}}$ & $\tilde{w}_{\mathrm{plnt}}$ & $\tilde{w}_{f}$ & $\tilde{w}_{\text {prox }}$ & $T$ & $\delta_{\mathrm{pl}}$ \\
\hline $7 a$ & 1073 & 2048 & 0.4 & & 2 & 1 & & 10 & & & .1 & no & & 870 & 1799 & .0004 & & & & & .0004 \\
\hline $7 \mathrm{c}$ & "' & $\prime \prime$ & 3.7 & & & 1 & 10 & 10 & .2 & & .05 & yes & $40^{\circ}$ & 767 & 1602 & .01 & 20 & 1 & 1 & .2 & .006 \\
\hline $7 d$ & 561 & 1024 & 1.0 & & & 1 & 10 & & & 20 & .1 & yes & $40^{\circ}$ & 767 & 1602 & .001 & & & & & .001 \\
\hline 11.2 & 5087 & 9182 & 11 & 200 & 2 & 1 & 10 & 1 & .01 & & & no & & 4332 & 9276 & .01 & & & & & .01 \\
\hline 9 & 2633 & 5120 & 29 & & & 1 & 10 & 1 & .1 & & & yes & $60^{\circ}$ & 4208 & 8561 & .03 & 20 & 1 & 1 & 2 & .017 \\
\hline 13 & 4290 & 8282 & 17 & & 2 & 1 & 10 & .1 & & & 5000 & no & & 55502 & 111653 & .002 & & & & & .002 \\
\hline
\end{tabular}

Figure 12: Optimization details for our examples: time $T$ in seconds, number of variables, weights, information if constraints $c_{\text {hyp }}, c_{\text {ang }}$ are used, quality of torsion-free support structure after remeshing, and quality after optimization. The measure of

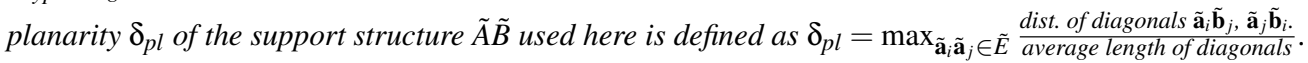

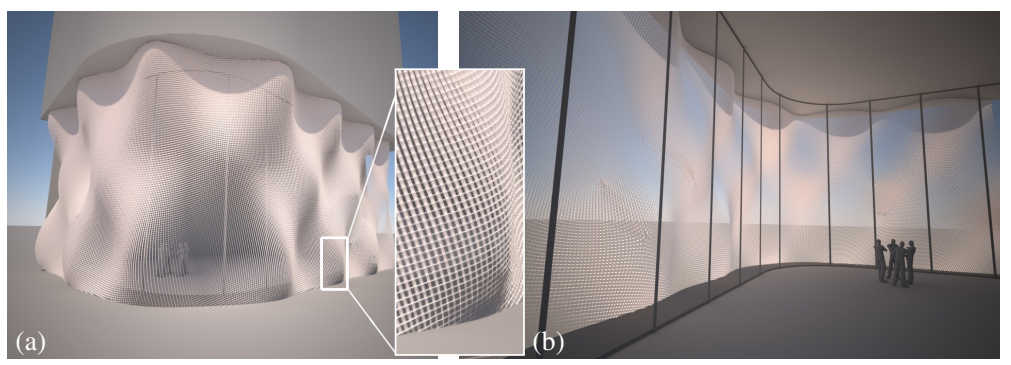

Figure 13: (a) A surface equipped with a torsion-free support structure effecting indirect lighting by reflecting sunlight onto the ceiling (Fig. 1, left). (b) If made from a non-reflecting material, shading and diffuse reflecting lighting is achieved. The screen is almost transparent when viewed from the inside ("veil of light").

with a focus on shading and lighting systems. Our procedures can be applied to any kind of geometry, from flat to double-curved. We thus combine an area deeply rooted in graphics (i.e., shading and lighting) with geometric computing and optimization in architectural design. Directions for future research are many: discrete curvatures defined in terms of normal congruences are a topic of discrete differential geometry. Other topics have to do with manufacturing and assembly, e.g. beams of constant height, more general shapes as shading elements etc.

Acknowledgments. This research was supported by the DFG Collaborative Research Center TRR 109, 'Discretization in Geometry and Dynamics' through grants I705-N26 and I706-N26 of the Austrian Science Fund (FWF).

\section{References}

[BS09] Bobenko A., SURIS Yu.: Discrete Differential Geometry: Integrable Structure. American Math. Soc., 2009.

[BZK09] Bommes D., Zimmer H., Kobbelt L.: Mixedinteger quadrangulation. ACM Trans. Graphics 28, 3 (2009), \#77, 1-10. doi:10.1145/1576246.1531383.

[DSM00] Doliwa A., Santini P., Mañas M.: Transformations of quadrilateral lattices. J. Math. Phys. 41 (2000), 944-990. doi:10.1063/1.533175.

[GR98] Ge Q. J., RAVANI B.: Geometric design of rational Bezier line congruences and ruled surfaces using line geometry. Computing [Suppl.] 13 (1998), 101-120. doi:10.1007/ 978-3-7091-6444-0_9.

[KEN*12] Kiser T., Eigensatz M., NguYen M. M., BomPAS P., PAUlY M.: Architectural caustics - controlling light with geometry. In Adv. Architectural Geometry 2012. pp. 91106. doi:10.1007/978-3-7091-1251-9_7.

[LN89] Liu D. C., NocedAL J.: On the limited memory method for large scale optimization. Math. Prog. B 45 (1989), 503-528. doi:doi:10.1007/BF01589116.

[LPW*06] LiU Y., PotTMann H., Wallner J., Yang Y.-L., WANG W.: Geometric modeling with conical meshes and developable surfaces. ACM Trans. Graphics 25, 3 (2006), 681-689. doi:10.1145/1141911.1141941.

[LXW*11] LiU Y., Xu W., Wang J., Zhu L., Guo B., Chen F., WANG G.: General planar quadrilateral mesh design using conjugate direction field. ACM Trans. Graphics 30 (2011), \#140, 1-10. doi:10.1145/2024156.2024174.

[PLW*07] PotTmann H., LiU Y., Wallner J., Bobenko A., WANG W.: Geometry of multi-layer freeform structures for architecture. ACM Trans. Graphics 26, 3 (2007), \#65,1-11. doi:10.1145/1275808.1276458.

[Por94] PORTEOUS I. R.: Geometric Differentiation for the Intelligence of Curves and Surfaces. Cambridge Univ. Press, 1994.

[PSB*08] Pottmann H., Schiftner A., Bo P., SchmiedHOFER H., WANG W., BALDASSINI N., WALLNER J.: Freeform surfaces from single curved panels. ACM Trans. Graphics 27, 3 (2008), \#76,1-10. doi:10.1145/1360612.1360675.

[PW01] Pottmann H., Wallner J.: Computational Line Geometry. Springer, 2001. doi:10.1007/978-3-64204018-4.

[PW08] PotTmann H., Wallner J.: The focal geometry of circular and conical meshes. Adv. Comp. Math 29 (2008), 249268. doi:10.1007/s10444-007-9045-4.

[YYG*07] Yu J., Yin X., Gu X., McMillan L., Gortler S.: Focal surfaces of discrete geometry. In Proc. SGP. 2007, pp. 23-32. doi:10.2312/SGP/SGP 07/023-032. 